\title{
Orbital and spin variability of the intermediate polar BG CMi
}

\author{
Y. G. Kim ${ }^{1,2}$, I. L. Andronov ${ }^{3,4,1}$, S. S. Park ${ }^{1}$, and Y.-B. Jeon ${ }^{5}$ \\ 1 University Observatory, Chungbuk National University, 361-763 Cheongju, Korea \\ 2 Institute for Basic Science Research, Chungbuk National University, 361 763, Korea \\ 3 Department of Astronomy, Odessa National University, T.G. Shevchenko Park, 65014 Odessa, Ukraine \\ e-mail: il-a@mail.ru \\ 4 Crimean Astrophysical Observatory, 98409 Nauchny, Ukraine \\ 5 Korea Astronomy Observatory and Space Science Institute, Daejeon 305-348, Korea
}

Received 4 March 2005 / Accepted 10 June 2005

\begin{abstract}
Results of a CCD study of the variability of the cataclysmic variable BG CMi obtained at the Korean $1.8 \mathrm{~m}$ telescope in 2002-2005 are presented. The "multi-comparison star" method had been applied for better accuracy estimates. The linear ephemeris based on 19 mean maxima for 2002-2005 is HJD 2453 105.31448(6)+0.01057257716(198)(E-764707). The period differs from that predicted by the quadratic ephemeris by Pych et al. (1996, AcA, 46, 279) leading to a possible cycle miscount. The statistically optimal ephemeris is a fourth-order polynomial, as a quadratic or even a cubic ephemeris leads to unaceptably large residuals: Min.HJD $=2445020.28095(28)+0.0105729609(57) E-1.58(32) \times 10^{-13} E^{2}-5.81(64) \times 10^{-19} E^{3}+4.92(41) \times$ $10^{-25} E^{4}$. Thus the rate of the spin-up of the white dwarf is decreasing. An alternative explanation is that the spin-up has been stopped during recent years. The deviations between the amplitudes of the spin variability in $V$ and $R$, as well as between phases are not statistically significant. However, the orbital light curves exhibit distinct difference; the corresponding color index shows a nearly sinusoidal shape with a maximum at orbital phase $\sim 0.2$. The variations of the amplitude of spin waves shows a short maximum at the phase of the orbital dip. The corrected ephemeris for orbital minima is Min.HJD $=2448368.7225(12)+$ $0.13474841(6) \cdot(E-24849)$ with a narrow dip occuring $0.07 \mathrm{P}$ later. The rate of the spin period variation seems to be changed, justifying the necessity of regular observations of intermediate polars.
\end{abstract}

Key words. accretion, accretion disks - stars: individual: BG CMi - stars: individual: FO Aqr - stars: binaries: close stars: novae, cataclysmic variables - stars: variables: general

\section{Introduction}

The object 3A $0729+103$ has been identified as an intermediate polar-type cataclysmic variable by McHardy et al. (1984). They found night-to-night variations of the mean brightness with an amplitude up to 0.22 in their sample. The orbital period was found to be $3^{\mathrm{h}} 14 \mathrm{~m} .1$ and maximal amplitude up to 0.15 . However, the most striking feature is the $15.2 \mathrm{~min}$ periodic variations of variable shape, which may be interpreted as the spin period of a magnetic white dwarf. In the General Catalogue of Variable Stars, the designation of the star is $\mathrm{BG} \mathrm{CMi}$.

The general model for intermediate polars is a red dwarf filling its Roche lobe, and a white dwarf, the magnetic field of which is strong enough to disrupt accretion disk completely or at least in its internal parts (cf. Patterson 1994; Warner 1995; Hellier 2001). Norton et al. (1992) suggested that the $913 \mathrm{~s}$ period is a spin-orbital beat period, thus indicating a weak or absent accretion disk and a fast flipping of the stream from one active pole to another.
Patterson \& Thomas (1993) suggested the double period of $1827 \mathrm{~s}$ instead of the observed $913 \mathrm{~s}$. Such a model corresponds to nearly equal accreting columns lying near the equatorial plane.

More recently, de Martino et al. (1995) concluded that this period is the spin one. From a Doppler tomogram analysis, Hellier (1999) reported on a "weak spin wave" with the same period.

The circular polarization has been detected by Penning et al. (1986). However, no evidence on its modulation with spin period has been detected, contrary to e.g. V405 Aqr (Shakhovskoy \& Kolesnikov 1997), which could justify the suggested spin period value.

The 15 min variations show a drastic period decrease, as was originally found by Augusteijn et al. (1991) and Singh et al. (1991), and studied in more detail by Patterson \& Thomas (1993) and Pych et al. (1996). Garlick et al. (1994) even suggested a cubic term in the ephemeris.

The study of rotational evolution of the white dwarf needs continuous monitoring of such objects. In the present paper we 
Table 1. Journal of observations: begin and end of the run, the number of observations $n$, the mean values $\langle m\rangle$ and rms deviations of the mean $\sigma_{m}$ and the filter.

\begin{tabular}{lcrrcc}
\hline \hline$t_{\text {start }}, 24 \ldots . .$. & $t_{\text {end }}, 24 \ldots .$. & $n$ & $\langle m\rangle$ & $\sigma_{m}$ & Filter \\
\hline 52635.2721 & 52635.3388 & 95 & 15.052 & 0.147 & $V$ \\
52638.2627 & 52638.3379 & 177 & 14.941 & 0.137 & $V$ \\
53035.0390 & 53035.1655 & 217 & 15.042 & 0.176 & $V$ \\
53036.1058 & 53036.2677 & 278 & 14.953 & 0.163 & $V$ \\
53052.9374 & 53053.1193 & 299 & 15.023 & 0.171 & $V$ \\
53383.0829 & 53383.1145 & 18 & 15.046 & 0.147 & $V$ \\
53383.0838 & 53383.1153 & 17 & 2.800 & 0.149 & $R$ \\
53384.1352 & 53384.1648 & 14 & 14.914 & 0.173 & $V$ \\
53384.1365 & 53384.1657 & 14 & 2.678 & 0.171 & $R$ \\
53385.9945 & 53386.1484 & 66 & 15.049 & 0.193 & $V$ \\
53385.9955 & 53386.1494 & 69 & 2.811 & 0.184 & $R$ \\
53404.9714 & 53405.1264 & 72 & 15.077 & 0.206 & $V$ \\
53404.9726 & 53405.1250 & 70 & 2.848 & 0.197 & $R$ \\
52635.2721 & 53405.1264 & 1236 & 15.005 & 0.173 & all $V$ \\
53383.0838 & 53405.1250 & 170 & 2.814 & 0.189 & all $R$ \\
\hline
\end{tabular}

report on further studies of the variability of the star based on the CCD observations obtained in the $V$ and $R$ filters at the $1.8 \mathrm{~m}$ telescope in 2002-2005.

\section{Observations and comparison stars}

The observations have been obtained with a thinned SITe $2 \mathrm{k}$ CCD camera attached to the $1.8 \mathrm{~m}$ telescope of the Korea Astronomy Observatory and Space Science Institute (Bohyunsan). The instrumental $V$ and $R$ systems have been used. To determine instrumental magnitudes of stars, the IRAF/DAOPHOT package (Massey \& Davis 1992) has been used.

Altogether, $1236 \mathrm{~V}$ and $170 \mathrm{R}$ observations have been obtained during 9 nights from December 26, 2002 to February 3, 2005 (JD $2452635-3405$ ) with an integration time $15^{\mathrm{s}}$ in 2002 and $30^{\mathrm{s}}$ in 2004 and 2005.

The journal of observations is presented in Table 1. In an addition to the mean magnitudes $\langle m\rangle$ in the instrumental system $m$ (see description below), we list the rms deviations $\sigma_{m}$ from the mean as an estimate of the characteristic total amplitude consisting of the variability at a few time scales and of the observational noise.

For further analysis of the individual runs, we mark them by the filter letter and five last digits of the integer part of the Helicentric Julian Date (HJD) of the beginning of the run.

\subsection{Using few comparison stars}

To obtain better accuracy, we have used few comparison stars in the field. Their $B V$ magnitudes have been estimated by Henden and Honeycutt (1995).

The procedure of the "artificial" mean weighted star has been used, which has been described by Andronov \& Baklanov (2004) and applied to the comparison stars in the vicinity of BG CMi by Kim et al. (2004). The instrumental magnitudes of all comparison stars have been checked, and the outstanding values have been excluded from the analysis. From the

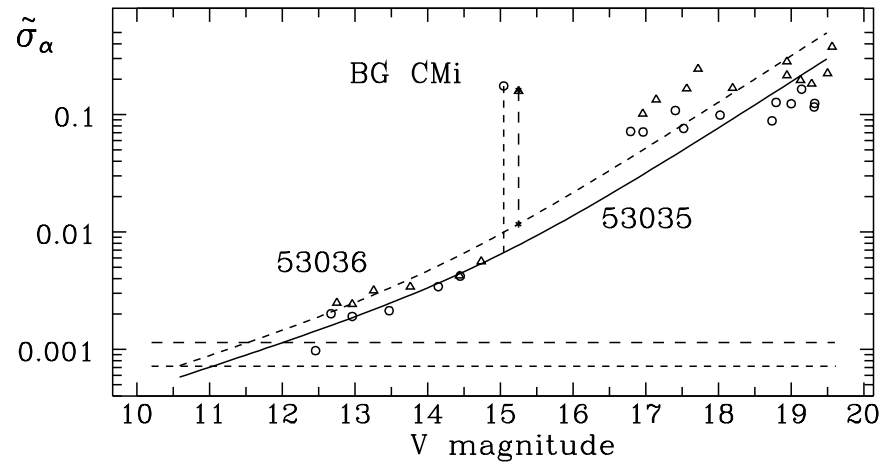

Fig. 1. The dependence on brightness of the rms deviation of stellar magnitudes of the variable and the comparison stars on the corresponding sample mean values. Open circles - for the night 53035 with relatively good weather, open triangles - for the night 53026 with highly variable atmospheric transparency. The curved lines correspond to the best fit assuming the Poisson noise for the counts of the star and background. Horizontal lines correspond to the estimated accuracy of the "artificial" mean weighted comparison star. The bottom curve in these two pairs correspond to better atmospheric conditions and thus smaller error estimates. Please note that the brightness is calibrated using results from the night 53035 , thus the points for the night 53036 with worse weather are shifted towards larger apparent magnitude and larger error estimates. Vertical lines project mean magnitude of BG CMi onto corresponding "accuracy-brightness" dependence.

images with all "good" measurements of the comparison stars, the mean magnitude differences have been computed for all comparison stars, then the magnitudes of all comparison stars have been determined using the magnitude of the "main" comparison star "05" (Henden \& Honeycutt 1995) $V=12 \mathrm{~m} .457$, $B-V=0$. 707. This star is also marked as "Comp 1" by Pych et al. (1996). Then the weights have been determined for each comparison star using iterations as described by Kim et al. (2004). Then, for each image, the brightness of the comparison star has been determined as the mean weighted value from estimates based on the magnitude differences between the variable and all comparison stars.

In Fig. 1, the "accuracy-magnitude" dependence is shown for two nights with "good" and "worse" atmospheric conditions. The corresponding error estimates for the "artificial" comparison star are 0.00072 and 0.00114 . The error estimates of the variable star itself for these nights may be estimated from a best non-linear fit as 0.0067 and 0.0117 , respectively.

The observed characteristics of 17 comparison stars are listed in Kim et al. (2004), the corresponding finding chart is available at http://uavso.pochta.ru/bgcmi_2.GIF. The differences between our instrumental magnitudes $V_{\text {in }}$ and the standard ones $V_{\mathrm{HH}}$ (Henden \& Honeycutt 1995) are well explained by the color reduction formula

$V_{\text {in }}=V_{\mathrm{HH}}-0.0025(16)+0.161(11) \Delta(V-R)_{\text {in }}$

(Kim et al. 2004). However, for our photometry, we have not taken into account the difference between our instrumental $V R$ systems and the standard ones, as there are no published estimates of the $R$ magnitudes of the comparison stars. The amplitudes will be expressed in the instrumental systems without any conversion. 

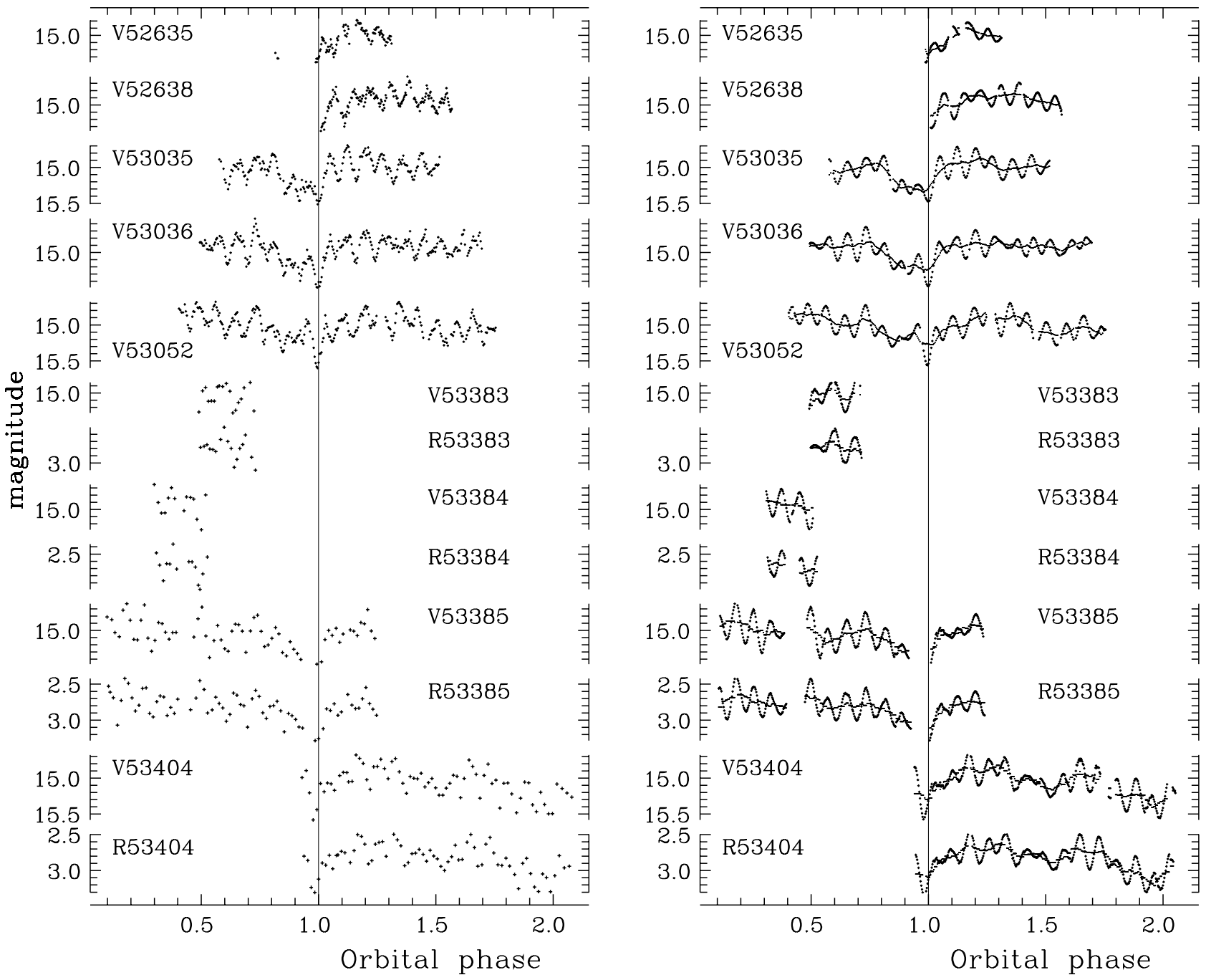

Fig. 2. The brightness variations of BG CMi during our observations with orbital phase computed using the ephemeris (4) by Patterson \& Thomas (1993). Left: original data; right: "running sine" fit $X_{\mathrm{RS}}(t, t, \Delta t)$ for times of observations and corresponding local mean $a(t, t, \Delta t)$. In the fits, some points have been skipped because of large error estimates of the smoothing value $(>0 \mathrm{~m} \cdot 10)$ near the gaps of the observations.

The light curves obtained for $9 \mathrm{~V}$ runs and $4 R$ runs are shown in Fig. 2. They exhibit both orbital and spin periodicity, as well as cycle-to-cycle variability, which will be studied below.

\section{Separation of spin and orbital variability}

\section{1. "Running sine" fit}

The orbital variations show a wave with an asymmetric minimum, which was interpreted as a grazing eclipse, when the accretion structure is partially eclipsed, but the white dwarf is not (cf. Hellier 1999, 2001). The initial ephemeris by McHardy et al. (1984) has been revised by Augusteijn et al. (1991) and Patterson \& Thomas (1993). Pych et al. (1993) have added 5 minima timings and corrected this revised ephemeris. Patterson \& Thomas (1993) distingush the orbital dips, which are close in phase to the $\mathrm{X}$-ray dips, and the orbital minima approximately corresponding to the mean between the descending and ascending branches of the eclipse at it's middle parts. The dip occurs $\sim 0.12 P$ after the "mid-eclipse", as one may see in their Fig. 6. To study the shape of the orbital curve, they subtracted the best fitting sinusoid with the spin period for each night.

To take into account the variability of the shape of the 913-sec signal, a local sinusoidal fit

$X_{\mathrm{RS}}\left(t, t_{0}, \Delta t\right)=a-r \cdot \cos \left(\omega\left(t-T_{0}\right)-2 \pi \varphi_{0}\right)$,

was applied. Here the data at time interval $\left(\left(t_{0}-\Delta t\right),\left(t_{0}+\Delta t\right)\right)$ are taken into account for least squares computation of the coefficients. The parameters $a$ (local mean value), $r$ (semi-amplitude) and $\varphi_{0}$ (phase of maximum in units of the short period) are dependent both on the mid-time $t_{0}$ of local sub-interval and the filter half-width $\Delta t$. Here $\omega=2 \pi / P, P$ is period, and $T_{0}$ is the initial epoch. To follow cycle-to cycle changes, we have adopted the value $\Delta t=0.5 P$.

General expressions for the statistical properties of running approximations for arbitrary basic and weight functions have been presented by Andronov (1997), with a comparative study of "running parabola", "running sine" and wavelet fits for various weight functions (Andronov 1999). This method had been 
Table 2. Characteristics of the multi-sinusoidal fit of individual runs of observations of BG CMi. The flux is expressed in units of $10^{-15} \mathrm{erg} \mathrm{s}^{-1} \mathrm{~cm}^{-2} \AA^{-1}$. The calibration in $V$ has been made using the magnitude of the comparison star. For $R$, the standard magnitude is unknown and has been arbitrarily set to zero. This makes values of intensity to be apparently large. They may be calibrated after further determination of the $R$ magnitude of the comparison star. For bad phase distribution caused by an incomplete coverage of the period in the first two runs, the values are highly biased and should not be taken for further analysis. Such biased or statistically insignificant values are marked by a semi-column “:”.

\begin{tabular}{cccccccc}
\hline \hline Date & $I_{0}$ & $R_{1}$ & $R_{2}$ & $R_{3}$ & $R_{4}$ & $R_{5}$ & $R_{6}$ \\
\hline$V 52635$ & $-759: \pm 129$ & $1381: \pm 2318$ & $1018: \pm 1670$ & $599: \pm 945$ & $270: \pm 400$ & $85: \pm 115$ & $14: \pm 17$ \\
$V 52638$ & $46: \pm 49$ & $77: \pm 89$ & $60: \pm 67$ & $39: \pm 41$ & $20: \pm 20$ & $7.17: \pm 6.67$ & $1.39: \pm 1.29$ \\
$V 53035$ & $3.527 \pm 0.042$ & $0.294 \pm 0.055$ & $0.337 \pm 0.054$ & $0.273 \pm 0.055$ & $0.071: \pm 0.059$ & $0.050: \pm 0.059$ & $0.092: \pm 0.056$ \\
$V 53036$ & $3.796 \pm 0.035$ & $0.362 \pm 0.050$ & $0.343 \pm 0.049$ & $0.167 \pm 0.050$ & $0.040: \pm 0.049$ & $0.075: \pm 0.049$ & $0.062: \pm 0.049$ \\
$V 53052$ & $3.578 \pm 0.035$ & $0.442 \pm 0.051$ & $0.179 \pm 0.050$ & $0.048: \pm 0.051$ & $0.068: \pm 0.049$ & $0.054: \pm 0.047$ & $0.097: \pm 0.048$ \\
$V 53385$ & $3.467 \pm 0.100$ & $0.257: \pm 0.158$ & $0.296: \pm 0.158$ & $0.296: \pm 0.153$ & $0.131: \pm 0.152$ & $0.059: \pm 0.136$ & $0.011: \pm 0.143$ \\
$R 53385$ & $272816 \pm 6747$ & $31239 \pm 10432$ & $17926: \pm 10145$ & $20531: \pm 9874$ & $4393: \pm 9968$ & $6942: \pm 9139$ & $1100: \pm 9558$ \\
$V 53404$ & $3.494 \pm 0.086$ & $0.406 \pm 0.124$ & $0.421 \pm 0.120$ & $0.072: \pm 0.121$ & $0.135: \pm 0.121$ & $0.100: \pm 0.120$ & $0.075: \pm 0.120$ \\
$R 53404$ & $272413 \pm 6526$ & $31228 \pm 9288$ & $32071 \pm 9066$ & $7256: \pm 9077$ & $6246: \pm 9273$ & $5251: \pm 9189$ & $3509: \pm 9264$ \\
\hline
\end{tabular}

applied for symbiotic variables UV Aur (Chinarova 1998) and V1329 Cyg (Chochol et al. 1999), where shorter variations with variable shape and nearly constant period are superimposed on (generally aperiodic) long-term trends.

The most recent ephemeris

$\mathrm{HJD}_{\text {Max }}=2445020.2800+0.010572992 \cdot E$

(Pych et al. 1996) has been used for reference. The long-term variability of the period and thus the initial epoch discussed in next section is negligible for such a local fit, the fit obtained for all interval of observations. Thus this initial value of the period may be used for local fits.

As an approximation to the longer-term orbital light curve, we have used local values of $a\left(t_{0}, \Delta t\right)$ at times $t_{0}$ coinciding with times of real observations. The corresponding values are plotted versus the orbital phase computed using the ephemeris

$\mathrm{HJD}_{\text {Min }}=2445020.384+0.1347486 \cdot E$

(Patterson \& Thomas 1993). The rms value of accuracy estimate of $a$ is $\sigma_{a}=0$. 0147 , so total amplitude of variations reaches $22 \sigma_{a}$.

The corresponding fits for slow variability $a(t, t, \Delta t)$ and fast+slow variability $X_{\mathrm{RS}}(t, t, \Delta t)$ computed for times of observations, where these fits are available, are shown in the right part of Fig. 2. One may note a significant shift of the mideclipse as compared to the ephemeris (4).

From our observations, we have determined 3 moments of minima using the "running sine" approximation, which are listed in Table 4 (runs V53035-V53053). Unfortunately, other runs do not cover eclipses completely, thus this method was not usable. For those runs (52669-52673, 53386, 53404), we have applied another method based on multi-sinusoidal fits, which will be discussed below.

\subsection{Multi-sinusoidal fits}

De Martino et al. (1995) have applied a multi-sinusoidal fit assuming multi-harmonic shape of the orbital light curve with frequencies $\omega_{J}=j \Omega$, where $\Omega=1 / P$ is the frequency corresponding to the orbital period $P, j=1$..s. In addition, they took
Table 3. Characteristics of the multi-sinusoidal fit of individual runs of observations of BG CMi (continuation of Table 2).

\begin{tabular}{cccc}
\hline \hline$T_{07}-2400000$ & $\langle I\rangle$ & $R_{7}$ & $\Delta m$ \\
\hline$V 52635.32067 \pm 0.00047$ & 3.492 & $0.320: \pm 0.126$ & \\
$V 52638.30276 \pm 0.00022$ & 3.861 & $0.444 \pm 0.056$ & \\
$V 53035.10202 \pm 0.00020$ & 3.536 & $0.457 \pm 0.055$ & 0.46 \\
$V 53036.19093 \pm 0.00021$ & 3.832 & $0.385 \pm 0.048$ & $0 . \mathrm{m} 39$ \\
$V 53053.02230 \pm 0.00018$ & 3.597 & $0.433 \pm 0.047$ & 0.38 \\
$V 53383.09882 \pm 0.00777$ & 3.508 & $0.284: \pm 0.176$ & \\
$V 53384.14518 \pm 0.00057$ & 3.976 & $0.802: \pm 0.297$ & \\
$V 53386.06917 \pm 0.00037$ & 3.524 & $0.561 \pm 0.126$ & 0.45 \\
$V 53405.04680 \pm 0.00043$ & 3.440 & $0.467 \pm 0.117$ & 0.44 \\
$R 53383.09798 \pm 0.00078$ & 277750 & $34337 \pm 14845$ & \\
$R 53384.14560 \pm 0.00067$ & 311765 & $57053 \pm 22282$ & \\
$R 53386.06915 \pm 0.00036$ & 276381 & $41497 \pm 8917$ & 0.47 \\
$R 53405.04708 \pm 0.00044$ & 267748 & $34647 \pm 8946$ & 0.43 \\
\hline
\end{tabular}

into account the 913-s pulses with frequency $\omega_{s+1}$ assuming its nearly sinusoidal shape.

The mathematical model for this two-frequency process is

$I(t)=I_{0}+\sum_{j=1}^{s} A_{j} \cos \left(\omega_{j}\left(t-T_{0 j}\right)\right)$.

Here $I(t)$ is intensity (computed from the magnitudes) and $A_{j}$ are semi-amplitudes of variations, and $T_{0 j}$ are initial epochs of the maxima of the waves corresponding to frequencies $\omega_{j}$. We use fluxes instead of magnitudes, to make results comparable to those of de Martino et al. (1995). According to the calibration (cf. Johnson 1955; Allen 1973), the magnitude $V=15$. 00 corresponds to the value of the monochromatic flux $f_{\lambda}=10^{-14.44}=3.63 \times 10^{-15} \mathrm{erg} \mathrm{s}^{-1} \mathrm{~cm}^{-2} \AA^{-1}$.

Results of the fit for the value $s=6+1$ (for orbital and spin variability, respectively), which had been adopted by de Martino et al. (1995), are listed in Tables 2 and 3. It should be noted that the parameter $I_{0}$ generally differs from the sample mean value $\langle I\rangle$.

For two short runs 52635 and 52638, the distribution of observations in the orbital phase is bad, thus the values of the parameters are highly biased and thus have no physical meaning, despite the fit itself is being good enough. The values 
deviating from zero less than $3 \sigma$ are not statistically significant, and marked by the symbol ":" as "bad" ones. For the first run, all values are thus marked as "bad", despite the amplitude for the spin variability having a reasonable value. For other nights, the estimates of the mean semi-amplitude of the spin variations varied in the range $(0.38-0.46) \times 10^{-15} \mathrm{erg} \mathrm{s}^{-1} \mathrm{~cm}^{-2} \AA^{-1}$. This interval slightly exceeds the error estimate, indicating cycle-tocycle and thus night-to-night variability of this parameter.

One may note that the ratio $P_{\text {orb }} / P_{\text {spin }}=12.74$ is far from an integer value, thus the orbital light curve differs from that of the next cycle by a spin phase shift of a quarter of period.

For all nights, the amplitudes of the waves with the orbital period $R_{1}$, its first harmonic $R_{2}$ and the spin period $R_{7}$ are statistically significant. The value $R_{3}$ slightly exceeds $3 \sigma$ only for two nights. For other nights, as well as for higher harmonics of the orbital period, the amplitudes $R_{3}, R_{4}, R_{5}, R_{6}$ are not statistically significant, and generally should not be taken into account. However, we present results in Tables 2 and 3 for the same " $6+1$ " multi-sinusoidal model, as has been proposed by de Martino et al. (1995). The multi-sinusoidal fits for fluxes are shown in Fig. 3. The fits show the presence of some variations of the orbital light curve, if taking into account such a large number of harmonics.

We may conclude, that, for our $V R$ observations obtained in 2002-2005, the highest harmonics are not significant, contrary to the B observations of de Martino et al. (1995) obtained in 1983-1992. For firm conclusions, new multi-color observations of the object are needed.

The direct comparison of the fluxes between this work and that by de Martino et al. (1995) is not possible, as they have published their results in another photometric system $(B)$. The peak-to-peak amplitude (Table 3) of the multi-sinusoidal fit of the orbital variability for 5 runs, which completely covers the period, varies from 0.38 to $0^{\mathrm{m}} \cdot 46$ in $V$. However, for the same nights, the amplitudes in $V$ and $R$ are practically the same around 0.45 .

Despite a generally good agreement of the multi-sinusoidal fit with the observations, showing both a-sinusoidal variability of the orbital light curve and the spin pulses superimposed, there are some important differences to the "running sine" fits. In the "global" multi-sinusoidal model, the amplitude of the spin pulses is constant (in the "flux" representation), thus its apparent "magnitude" variability with phase is caused by the variability of the smoothed phase dependence of the orbital flux, and not by the physical variability of individual spin pulses.

\subsection{Night-to-night variability}

The mean brightness variations during 9 nights of our observations show only minor variations with a peak-to-peak amplitude of $0 \mathrm{~m} .10$, as one may see from the values of $I_{0}$ in Table 2 . This value is smaller than that 0.22 reported by McHardy et al. (1984). This may be an effect of a small sample. However, if assuming that these variations are more pronounced in the ultraviolet, and given that McHardy et al. (1984) have used unfiltered observations, one may interpret such a difference in amplitudes as a result of different photometric systems.

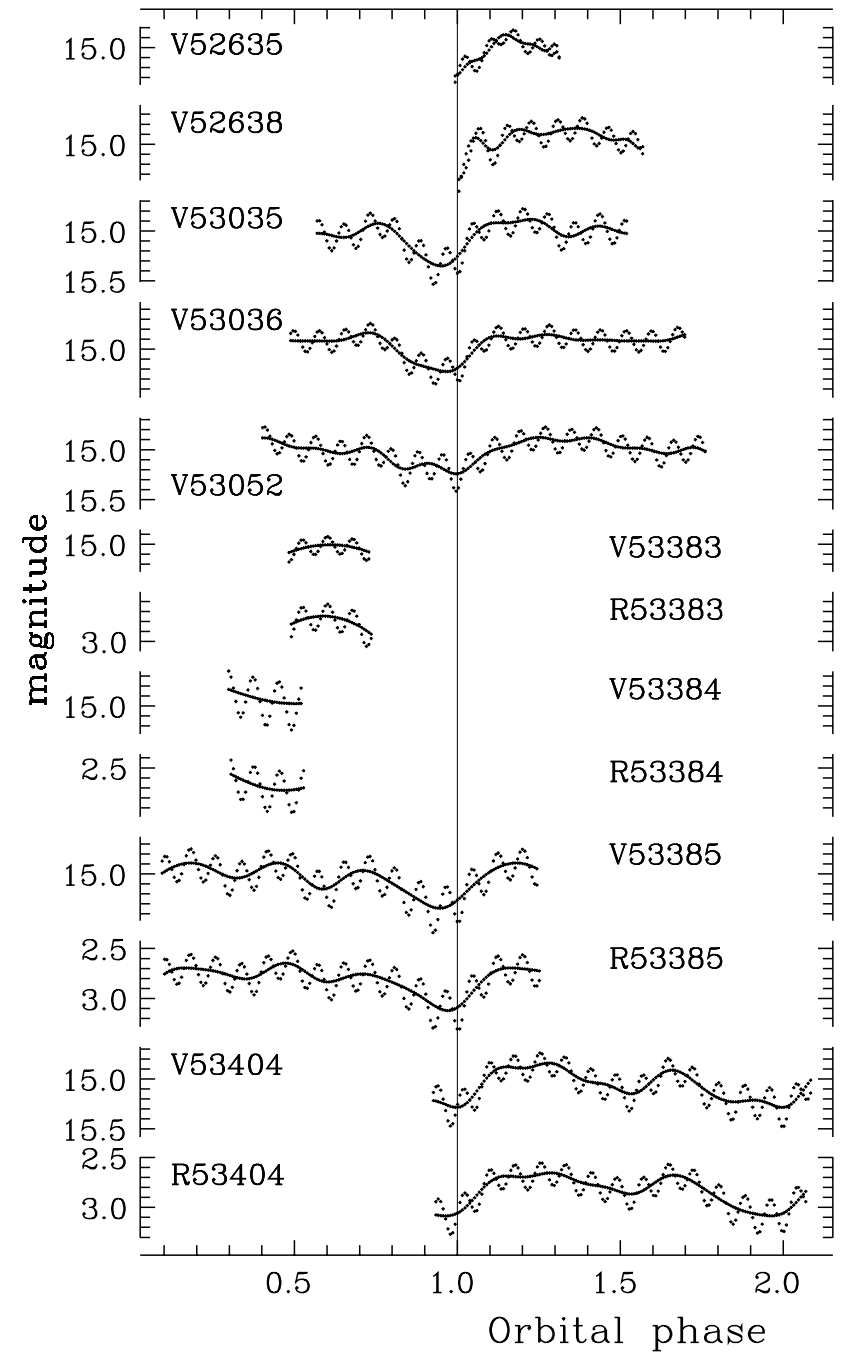

Fig. 3. The multi-sinusoidal fit for individual runs of observations of BG CMi. The orbital light curve is shown as an abridged sum $(s=6)$, whereas the "orbital+spin" variations are fitted by a complete sum $(s=7)$. The fits have been computed for fluxes, following de Martino et al. (1995), and converted to stellar magnitudes for comparison with the original data and the "running sine" fits. For $R$, the magnitude is expressed as the "Var-Comp" difference.

Unfortunately, we have not found other amplitude estimates in the literature, thus the question is still open.

\section{Color variability with the phase of orbital and spin period}

\subsection{Quasi-simultaneous VR observations}

Our observations in $V$ and $R$ have been made using alternatively changing filters. In this case, direct color measurements are not possible, as BG CMi shows fast variability. To make a set of quasi-simultaneous observations, the interpolating or smoothing technique is to be applied. For another cataclysmic variable TT Ari, we have used the method of "running parabolae" Andronov (1997) to make a set of simultaneous smoothed values in 3 colors $U B V$ (Tremko et al. 1996; 
Andronov et al. 1999). That method is effective for stars with flickering-dominated variability.

For BG CMi, the major amplitude of variations is due to orbital and spin variability, thus we have applied an interpolating procedure to determine brightness at missing times using times of observations in another filter. For the local sequence $V R V R V R V$, we have applied a local cubic polynomial fit using two nearby observations before and after. For regularly spaced observations, this will lead to an interpolating formula $\tilde{x}_{i}=$ $\left(9\left(x_{i-1}+x_{i+1}\right)-\left(x_{i-3}+x_{i+3}\right)\right) / 16$ assuming that indices $i-2, i+2$ correspond to the same channel as the point $i$. The statistical error of this smoothing value is $\sigma_{\tilde{x}}=(41 / 64)^{1 / 2} \sigma_{x} \approx 0.80 \sigma_{x}$, where $\sigma_{x}$ is an accuracy estimate of the individual signal value. Such an approximation is better than a linear one in our case of relatively fast oscillations. For real observations with nearly the same time interval between subsequent points, the weight coefficients have been computed according to a polynomial interpolation. Near the borders, where the point $i \mp 2$ is located outside a run of equidistantly spaced times, we have applied a parabolic fit using the points $i \mp 1, i \pm 1, i \pm 3$ or a linear interpolation using the observations $i \mp 1, i \pm 1$. No extrapolation has been made to avoid large errors.

As a result of this procedure (see Andronov \& Baklanov 2004 , for a detailed study of statistical properties of such approximations), we have obtained 269 pairs of "simultaneous" $V R$ observations, for which the instrumental color index $V-R$ has been computed. The data points which are present only for one color for a trial time, have been excluded.

As unfortunately the brightness of the comparison stars in $R$ is unknown, we cannot convert instrumental magnitudes and the corresponding color index to the standard system. Thus the magnitudes will be expressed as Var-Comp, and the colors will be represented with respect to the comparison star, i.e. our $V-$ $R=\left(V_{\text {var }}-V_{\text {comp }}\right)-\left(R_{\text {var }}-R_{\text {comp }}\right)$.

\subsection{Brightness and color variations}

The corresponding light and color curves for this set of "original+interpolated" points are shown in Fig. 4. Despite cycleto-cycle variability of the individual spin pulses, their characteristic times are much longer than the time resolution of our observations, thus the interpolation has not biased the shape of the light curve, as one may see by comparing with the data presented at high-harmonic fits for intensities at Fig. 3. Because of the relatively small amplitude of orbital variations $\left.(\sim 0)^{\mathrm{m}} 4\right)$, the difference in the fits of magnitudes and intensities is compared with the error estimates, and may only be important at the grazing eclipse and subsequent dip.

The degree of the trigonometric polynomial for the multisinusoidal fits have been reduced to $s=2+1$, taking into account that higher harmonics of the orbital variability are not statistically significant (see Table 2). Despite the long runs are presented in Fig. 4 separately, the fits have been obtained for a long joint run to decrease the night-to-night scatter. Thus the approximation of orbital variability is the same for both nights. The spin frequency is 94.58 cycles/day or 12.7447 cycles per orbital period, thus the phase of the spin variations changes

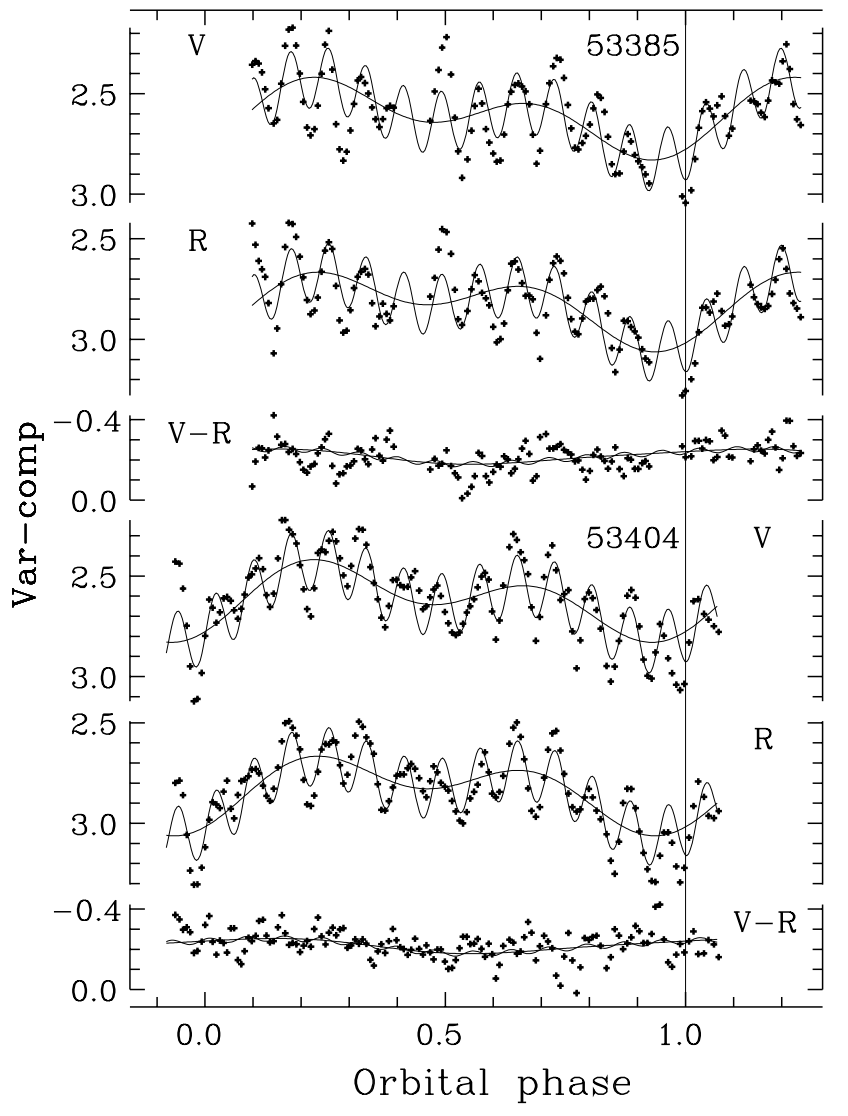

Fig. 4. Brightness and color variations for original and interpolated points (dots) and approximations of the orbital and "orbital+spin" variability.

by a quarter of a period every $P_{\text {orb }}$. However, in our observations, the interval between the orbital minima was apparently $141 P_{\text {orb }}=1797.003 P_{\text {spin }}$, so we have a rare event of coincidence of both spin and orbital phases in both nights, so the fits for the runs are the same.

Under such conditions, all differences between the curves are due to physical variability of the star and not to the effects of dependence on two independent phases. The high-amplitude spin pulsation cycle at $\varphi_{\text {orb }} \sim 0.5$ seen in the run 53385 has no counterpart for the run 53404, where in the vicinity of this phase an opposite phenomenon (amplitude decrease) have been observed.

The mean magnitudes with respect to the comparison star are $m_{0 V}=2.612(7)$ and $m_{0 R}=2$ m $830(6)$, so the relative color index is equal to $m_{0 V}-m_{0 R}=-0$. $218(4)$ and corresponds to a slightly larger temperature than the comparison star. This color difference is small enough to justify use of the star as the comparison one.

The orbital light curve has two minima separated by a halfperiod. If the phase zero corresponds to the grazing eclipse, the secondary minimum may be interpreted by a partial eclipse of the illuminated secondary by the disk and/or ellipticity of the disk. The semi-amplitudes of the contribution with the orbital period are $R_{1 V}=0.116(10) R_{1 R}=0.123(9)$, with nearly the same values for its harmonic: $R_{2 V}=0.119, R_{2 R}=0$. $114(8)$. 


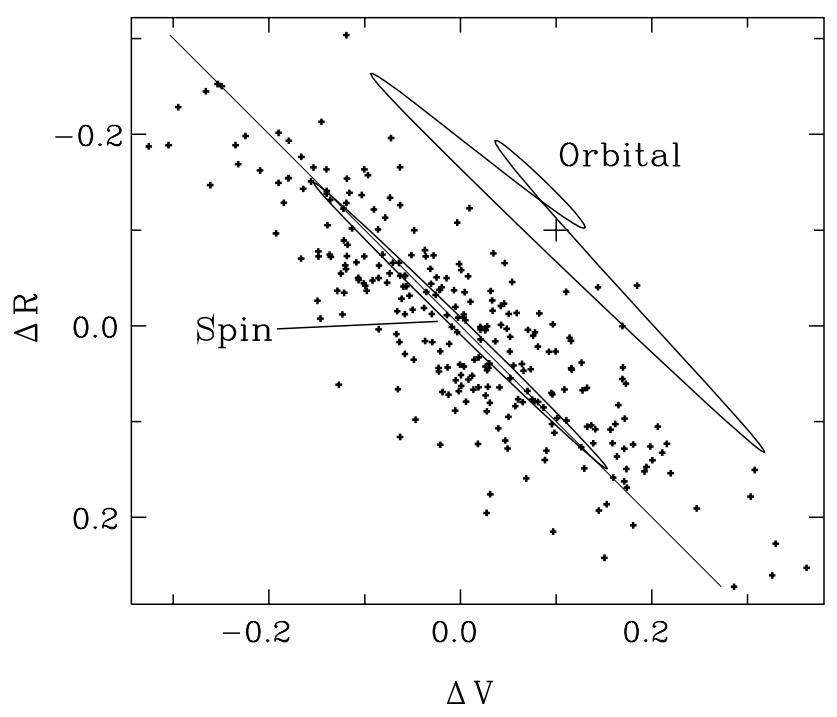

Fig. 5. Two-color diagram for the deviations of original and interpolated points from orbital fit (dots). The line corresponds to the unitary slope $\Delta R=\Delta V$. The closed lines correspond to the orbital fit (shifted by +0.1 in $V$ and -0.1 in $R$ ) and deviations of the "spin+orbital" fit from the pure "orbital" one. The orbital curve is shifted by $+0 .{ }^{\mathrm{m}} 1,-0^{\mathrm{m}}$. 1 for illustrating purposes. The cross indicates $\pm 1 \sigma$ values for accuracy of the smoothed curves in $V$ and $R$.

To determinine effective amplitudes in other types of cataclysmic variables with aperiodic light curves, Andronov et al. (1999) used the rms deviation $\sigma_{*}$ of the smoothed values from the mean. For periodic variables, one may use an effective semi-amplitude $R_{*}=\left(R_{1}^{2}+R_{2}^{2}\right)^{1 / 2}=2^{1 / 2} \sigma_{*}$. For our data, such values are practically identical for two colors: $R_{* V}=0.166(10)$ and $R_{* R}=0$. $167(9)$. Such behaviour of the intermediate polar BG CMi is intermediate between an increase of amplitude with decreasing wavelength in non-magnetic nova-like variables with superhumps (e.g. MV Lyr, Walker 1954; TT Ari, Andronov et al. 1999) and with eclipses (e.g. DW UMa, Ostrova et al. 2005) or with increasing wavelength in magnetic classical polars (e.g. AM Her, Szkody \& Brownlee 1977).

The multi-sinusoidal analysis of the color index shows that the amplitude corresponding to the orbital period $\left(R_{1}=\right.$ $0 \mathrm{~m} 036(6))$ is much larger than for other frequencies. The star becomes most blue at the orbital phase 0.42 and most red at 0.82 .

A similar situation is with the semi-amplitudes of the spin variations: $R_{3 V}=0 \mathrm{~m} \cdot 154(9)$ and $R_{3 R}=0 \mathrm{~m}$ 147(8), so the color excess of the spin variations $\Delta V-R=-2.5 \lg \left(R_{3 V} / R_{3 R}\right)=$ $-0 \mathrm{~m} 05(9)$ is zero within error estimates. Nearly the same similarity of the spin amplitudes is observed in another intermediate polar FO Aqr but with much smaller values $R_{3 V}=0$. 087(6) and $R_{3 R}=0$.095(4) (Andronov et al. 2005b).

\subsection{Two-color diagram}

The two-color diagram for the deviations of the data from the orbital fit is shown in Fig. 5. The line of equal shifts $\Delta R=\alpha \Delta V$ satisfactorily represents individual points with $\alpha=1$, and is in excellent agreement with the sinusoidal component. As there is a very small difference between the amplitudes discussed in previous section, the slope $\alpha$ seems to be slightly less than unity. However, this effect is within the error estimates. Also the phase shift between oscillations in $V$ and $R$ is not statistically significant, thus making the minor axis of the ellipse vanish. There is a scatter of individual points of 0.048 , which is much larger than the accuracy estimate of the individual measurement of the star of such brightness ( 0.01 , see Fig. 1). Such scatter may correspond to a possible flickering with much higher amplitude in $V$ than in $R$, that is usual for accretion. This hypothesis may be supported by a the result that the $V-R$ color is a much more high correlated with $V$ than with $R$. The corresponding correlation coefficients are $r[V-R, V]=0.5(5)$ and $r[V-R, R]=0.20(8)$, respectively. This may be also the result of the contribution of "strongly colored" spin cycles, when the color variations distinctly deviate from the smooth orbital curve. However, based on the present material, it is not possible to obtain sufficient statistics on the occurence of "colored" and "non-colored" pulses.

Optical, UV and X-ray fluxes of intermediate polars show highly structered accretion geometry (cf. Kim \& Beuermann 1995, 1996). Our results can be used to model such an accretion flow.

The difference in the shape of the orbital light curves in two colors leads to a change of the trajectory at the two-color diagram from a line to an "8-like" figure. Such difference in behaviour is the observational basis for future theoretic modeling of the orbital variability.

\subsection{Periodogram analysis}

To study differences in light curves in different colors, we have applied the periodogram analysis to the deviations of the individual data points from the "orbital" $\mathrm{O}-\mathrm{C}_{\text {orb }}$ and "orbital+spin" $\mathrm{O}-\mathrm{C}_{\mathrm{orb}+\text { spin }}$ fits. For some objects, there are peaks present at beat frequencies, which are the combinations of the orbital frequency $\Omega$ and the spin frequency $\Omega$ (cf. Warner 1986 1995; Wynn \& King 1992; Patterson 1994; Hellier 2001).

The test-function $S(f)$ has been used (see Andronov 1994, for details). The deviations $\mathrm{O}-\mathrm{C}_{\text {orb }}$ show the series of strong peaks corresponding to the spin frequency and its 19-day aliases (the interval between the runs 53404 and 53 385). The peaks near this frequency practically disappear for $\mathrm{O}-\mathrm{C}_{\text {orb+spin }}$, and there is no evidence for the spin frequency for the color index for both types of deviations. However, there are strong peaks near $3 \Omega$ (but not exactly "at"). Also in $R$ there is an apparent peak at $\sim 55$ cycles/day. We suggest that these apparent peaks arise from instabilities of the light curves. Such quasiperiodic oscillations with a time-scale of 15-60 min are characteristic for some cataclysmic variables of similar orbital periods (e.g. Tremko et al. 1996; Andronov et al. 1999). However, our periodograms show no significant peaks at frequences, where the peaks have been found in previous years by Patterson \& Thomas (1993) and Hellier (1999). There is no evidence for significant peaks at the beat/multiple frequencies, where such peaks are seen in other cataclysmic variables. 


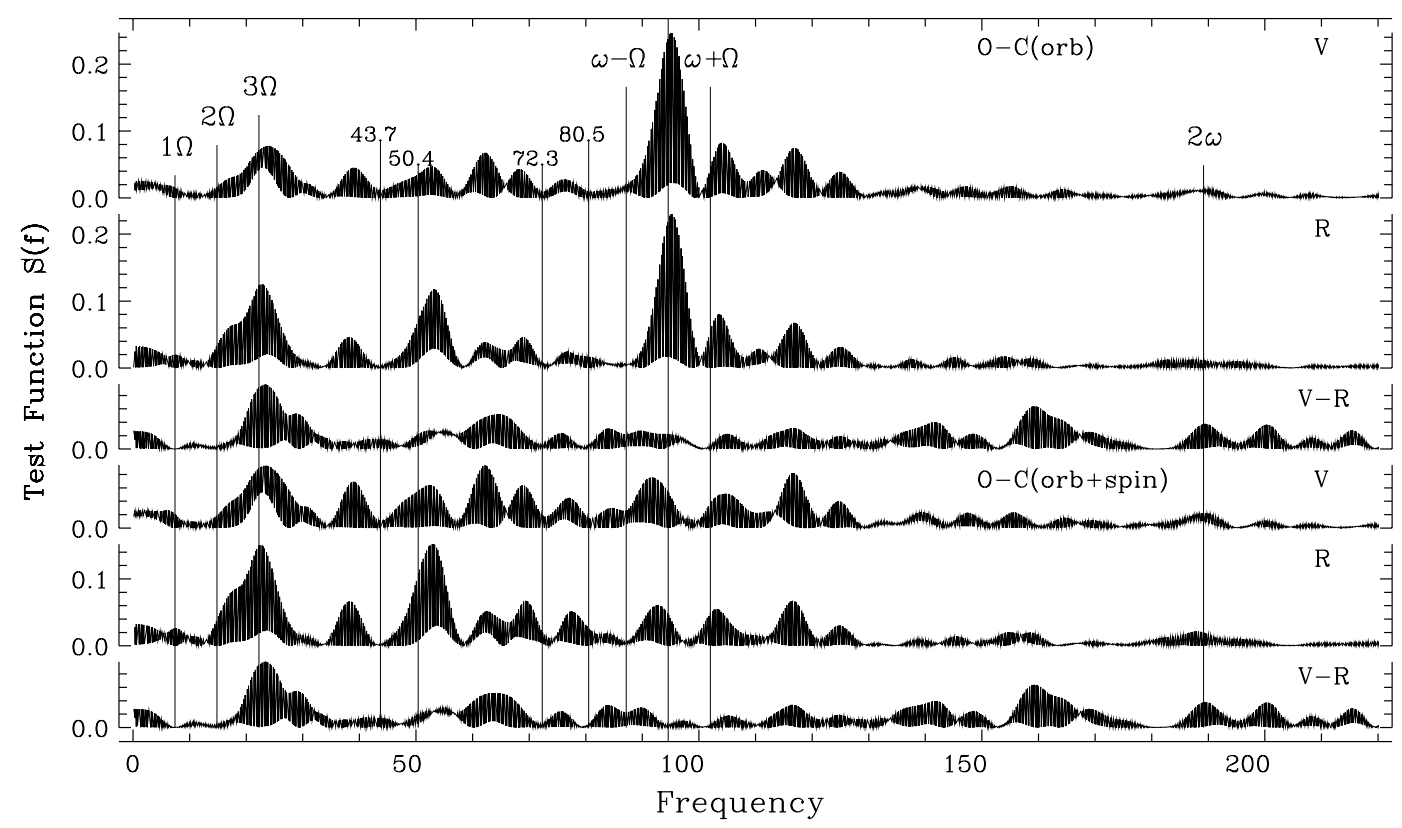

Fig. 6. Periodograms $S(f)$ for deviations of the brightness and color from the orbital fits for two nights of two-color photometry. Vertical lines mark positions of the orbital $(\Omega)$ frequency and its harmonics, as well as of the spin $(\omega)$ frequency and its harmonic $(2 \omega)$ and sidebands $(\omega \pm \Omega)$. Numbers correspond to the frequencies suspected by Hellier (1999) and Paterson \& Thomas (1993).

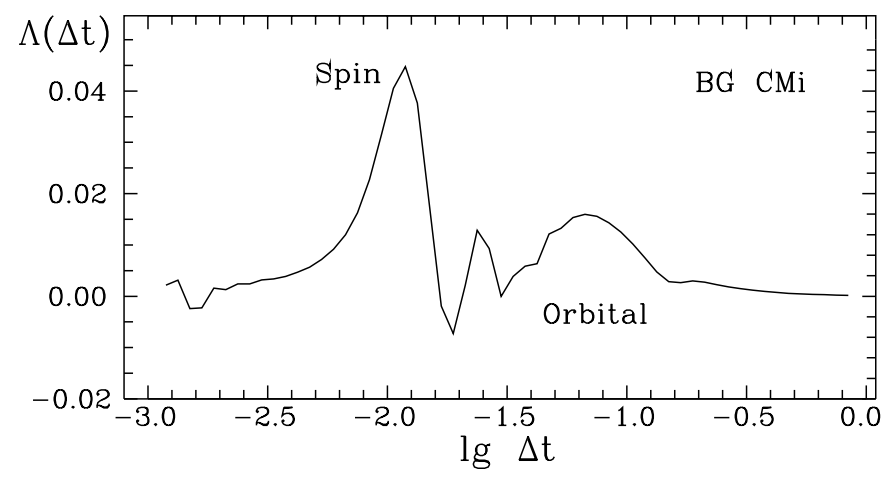

Fig. 7. The dependence of the test function $\Lambda(\Delta t)$ on the filter halfwidth $\Delta t$. The two highest peaks correspond to the spin and the harmonic of the orbital variability.

\subsection{Scalegram analysis}

The scalegram analysis has been carried out using the "running parabola" approximation. The " $\sigma(\Delta t)$ " scalegram (Andronov 1997) shows a shape characteristic for a two-component signal. For such a case, is useful the " $\Lambda(\Delta t)$ " scalegram (Andronov 2003), which is shown in Fig. 7 for 2002-2004, when onecolor photometry provided better time resolution. The most prominent maximum corresponds to the spin variability with an effective period of $P_{\text {spin,eff }}=0.01058$ and semi-amplitude $r_{\text {spin,eff }}=0$. 139 . This value slightly exceeds the mean semiamplitude of the spin variations obtained from multi-sinusoidal fits $r_{\mathrm{ms}, \text { eff }}=-2.5 \lg \left(\left(\left(I_{0}+I_{7}\right) /\left(I_{0}-I_{7}\right)\right)^{1 / 2}\right)$, which is equal to 0.127 . This excess argues for more rapid variations of the individual cycles and their phase shifts. For a pure sinusoidal signal, both methods should give closer results.

The second peak at the " $\Lambda(\Delta t)$ " scalegram corresponds to the first harmonic of the orbital variability with $P_{2, \text { eff }}=0.061$ and $r_{2, \text { eff }}=0$. 083 . The third peak in between may correspond to the third harmonic of the orbital period, because the shape is not sinusoidal. As expected, the effective values for the spin period coincides much better with the values obtained by other methods than that for the orbital variability. This is caused either by the duration of observations comparable with the orbital period or by the non-sinusoidal shape of the orbital variability.

\section{194-minute orbital period}

\subsection{Orbital minima and dips}

The orbital minimum has a specific asymmetric shape at its bottom, showing a narrow dip occurring by $\sim 0.07 P$ later than the mid-eclipse, as one may see from Fig. 6 of Patterson \& Thomas (1993). To study further period behavior, we have used $42 \mathrm{~min}$ ima published in Table 1 of their paper. It seems that the one corresponding to HJD 2445730.443 seems to be a misprint. Following the authors, we have checked the original reference of Singh et al. (1991) for the orbital maximum and corrected the decimal part of this point to 0.120. Then we have collected 5 minima obtained by Pych et al. (1996). In this work, we have determined 3 moments of centers of minima from the function $a\left(t_{0}\right)$ obtained using the "running sine" fit for the nights $53035,53036,53052$, when the coverage of eclipses was good. The moments of dips for these nights have been determined as those corresponding to the minumum of $a\left(t_{0}\right)$. For the nights 53384,53404 , the coverage of eclipses is not complete, so we have used the multi-periodic fit $(s=6+1)$.

Additionally, we have analyzed 3 nights of unpublished unfiltered CCD photometry with a 120 -s integration time, which was obtained by Foote (2003) at the $93 \mathrm{~cm}$ telescope in Utah as a part of activity of the Center for Backyard Astrophysics (http://cba.phys. columbia. edu). From 3 nights, 4 mean 
Table 4. Moments of orbital minima and dips.

\begin{tabular}{|c|c|c|c|}
\hline Min BJD & $E$ & $\mathrm{O}-\mathrm{C}$ & Type \\
\hline$W 52669.9227$ & 56769 & -0.0046 & Mid-eclipse \\
\hline$W 52670.7231$ & 56775 & -0.0127 & -"- \\
\hline$W 52679.7595$ & 56797 & -0.0075 & -"- \\
\hline$W 52673.6927$ & 56842 & -0.0044 & $-"-$ \\
\hline$V 53035.0875$ & 59479 & -0.0085 & -"- \\
\hline$V 53036.1663$ & 59487 & -0.0077 & -"- \\
\hline$V 53053.0073$ & 59612 & -0.0102 & -"- \\
\hline V 53386.1087 & 62084 & -0.0074 & -"- \\
\hline$R 53386.1122$ & 62084 & -0.0039 & -"- \\
\hline$R 53404.9759$ & 62224 & -0.0050 & -"- \\
\hline$V 53405.1050$ & 62225 & -0.0106 & -"- \\
\hline$V 53035.1023$ & 59479 & 0.0063 & Orbital dip \\
\hline$V 53036.1724$ & 59487 & -0.0016 & -"- \\
\hline$V 53053.0170$ & 59612 & -0.0005 & -"- \\
\hline$V 53405.1152$ & 62225 & -0.0004 & -"- \\
\hline
\end{tabular}

minima have been determined using multi-sinusoidal " $(2+1)$ " fits, one of them (52673.6927) corresponds to the mean light curve for all these nights. This "white light" photometry is marked as "W" in the text.

All orbital minima determined in this work are listed in the Table 4.

Our minima show smaller scatter than that from the tables by Patterson \& Thomas (1993) and Pych et al. (1996), where the phases of minima range from -0.13 to +0.22 , because of the inhomogeneity of their collection of previously published (or even estimated from the light curves) minima. Adding our 11 points to their 47 minima, the best fit ephemeris has been obtained

$$
\begin{aligned}
& \text { Min.HJD }=2445020.3894+0.13474841 \cdot E \\
& \pm \quad 20 \pm 6 \\
& \text { Min.HJD }=2448368.7225+0.13474841 \cdot(E-24849) \\
& \pm \quad 12 \pm 6 \text {. }
\end{aligned}
$$

The second ephemeris corresponds to the minimum error estimate of the initial epoch, which is closest to a sample mean of times used.

From our minima timings, we may estimate phase difference between the mid-eclipse and orbital dip to range from 0.04 to $0.11 P$. The mean phase of our $7 V R$ minima with respect to the ephemeris of Patterson \& Thomas (1993) is $\left\langle\varphi_{\min }\right\rangle=$ $-0.056 \pm 0.007$; it reaches $8 \sigma$ and thus is to be taken into account. Similar negative O-C are seen for the "W" observations obtained by Foote (2003). According to the new ephemeris (6), the mean phase $\left\langle\varphi_{\min }\right\rangle=-0.012 \pm 0.007$ is much close to zero, but there is still some deviation. It may be explained by inhomegeneity of the previously published data, where both orbital minima and dips have been included without separation. The dips occur at the mean phase $\left\langle\varphi_{\text {dip }}\right\rangle=+0.049 \pm 0.013$, according to the ephemeris (6), so the mean phase difference is $\left\langle\varphi_{\text {dip }}\right\rangle-\left\langle\varphi_{\text {min }}\right\rangle=+0^{p} .062 \pm 0.015$. There is also a suggestion that the minima in $R$ occur by $\sim 0.034 \pm 0.007$ later than in $V$. However, it is based only on two nights of two-color observations, when the minima were badly covered by the observations, thus it may have resulted from statistical fluctuations

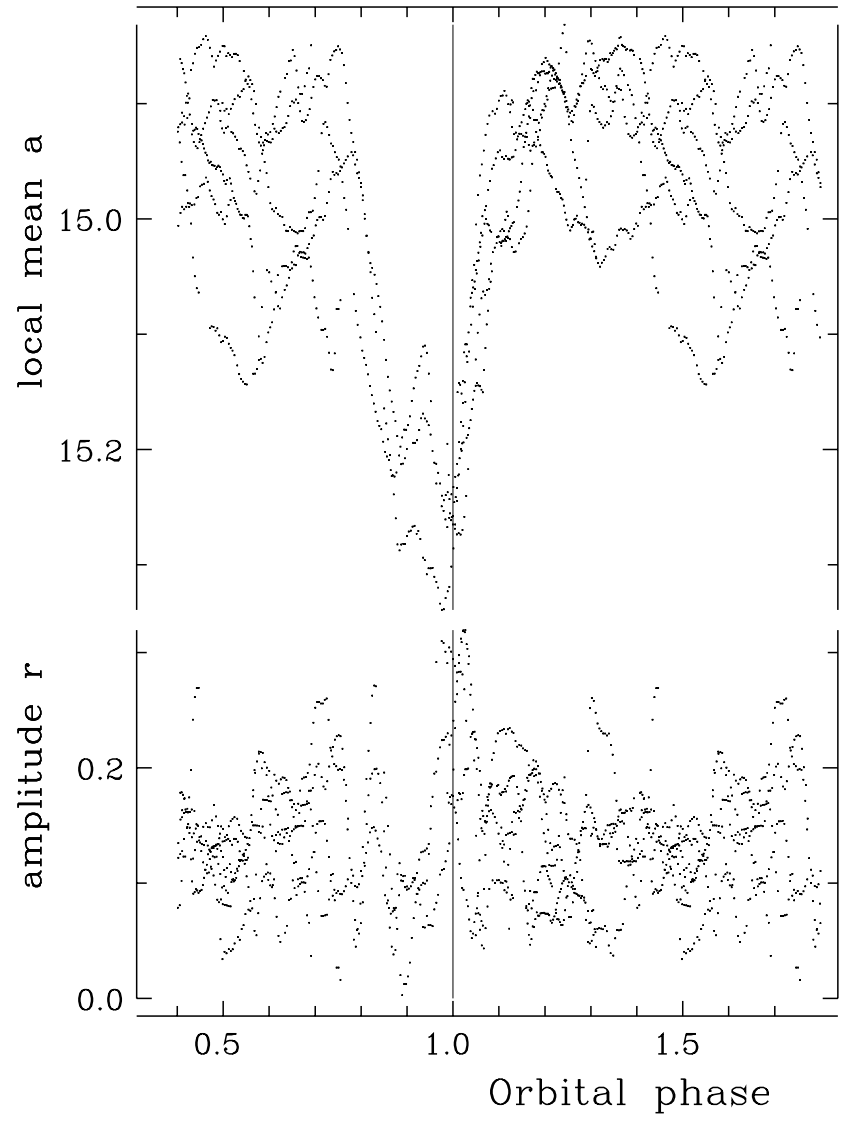

Fig. 8. The dependence on the orbital phase of the local mean and semi-amplitude of the spin variability.

of observational errors. This suggestion has to be checked in future studies.

The peak-to-peak amplitudes of the orbital variations, obtained from the multi-periodic fit, are 0.43 and 0.41 for the runs V 53385 and V 53404, respectively. For the filter $R$, these values are practically the same: 0.43 and 0.41 .

As the orbital periods of cataclysmic variables undergo evolutionary decrease (cf. Patterson 1984), we have checked the $\mathrm{O}-\mathrm{C}$ diagram for the presence of a quadratic term in an ephemeris. Unfortunately, the scatter of the early data compiled by Patterson \& Thomas (1993) is rather large (rms value of 0.069). So, despite much better accuracy of the data in their and our work and that by Pych et al. (1996), the obtained value of the $\dot{P}_{\text {orb }}$ deviates from zero only by $0.9 \sigma$, and thus the hypothesis of decrease of the orbital period may not yet be justified from all available data spanning more than two decades.

\subsection{Phase dependence of the pulse-averaged brightness and amplitude}

The characteristics of the "running sine" fits are shown in Fig. 8. The most important of them are the local mean (pulseaveraged brightness) $a(t)$ and semi-amplitude $r(t)$ of the spin pulse. The orbital variability is prominent with the broad $(0.2 P)$ minimum and the lagged dip. The amplitude shows night-tonight scatter being relatively constant at the phase intervals 
0.45-0.50. The phases have been computed according to the ephemeris (4) by Patterson \& Thomas (1993).

At the phases of orbital minimum, the semi-amplitude shows a minimum for all nights. However, the dip is characterized by an abrupt peak of the semi-amplitude. The peak lags the dip by $\approx 0.02 P$, that may be partially explained by an abrupt ascending branch after the dip causing an apparent increase of the amplitude. However, this phenomenon may not explain the peak completely, as the amplitude really increases, as one may check the original and smoothed light curves.

\section{Variability of the spin period}

\subsection{Determination of times of extrema}

For the analysis of one-color observations in 2002-2004, we have used the method of "asymptotic parabolae" (AP), proposed by Marsakova \& Andronov (1996) for timings of extrema of signals with cycle-to-cycle variability. The tables of 58 maxima and 63 minima have been published separately by Andronov et al. (2005a). They have used these results to obtain 5 nightly and 2 seasonal "mean" maxima timings with much better accuracy. The extrema timings show a scatter of phases within a relatively large "corridor" of \pm 0 ? 3 , except 3 points deviating from the mean value toward even larger values. These 3 points have been omitted. The mean deviation of the minima from the phase 0.5 corresponding to maxima is only $+0.003 \pm 0.020$ and thus is not statistically significant. This is in a contradiction to a qualitative suggestion of more abrupt brightness increase than decrease (de Martino et al. 1995).

For the observations obtained in 2005, the use of alternating filters had led to half the number of data per cycle, and thus it was not possible to apply the same method, which is effective to study characteristics of individual cycles. For determination of the individual extrema in this case, we have used the multi-sinusoidal fit (5) for "Var-Comp" magnitude differences. Because of the statistical symmetry of spin pulses, we have used only the wave with a spin period without any additional harmonics, contrary to fitting the shape of the orbital phase curve. These timings for earlier one-color observations are in good agreement with the results presented by Andronov et al. (2005a), but, for uniformity, we have used results obtained using the multi-sinusoidal fits.

The corresponding timings of the "mean" maxima are listed in Table 3. To obtain more accurate results, we have determined "mean" maximum for all 3 nights of the "W" observations, as well as for two long runs in $V$ and $R$.

\subsection{Models of variability}

The variability of the spin period of BG CMi had been detected by Singh et al. (1991) and Augusteijn et al. (1991). Patterson \& Thomas (1993) determined a quadratic ephemeris based on their own and compiled times of maxima. Garlick et al. (1994) suggested even a third-order polynomial to fit the $\mathrm{O}-\mathrm{C}$ diagram. In more recent study, Pych et al. (1996) rejected this suggestion, arguing for a sufficience of the second-order polynomial.

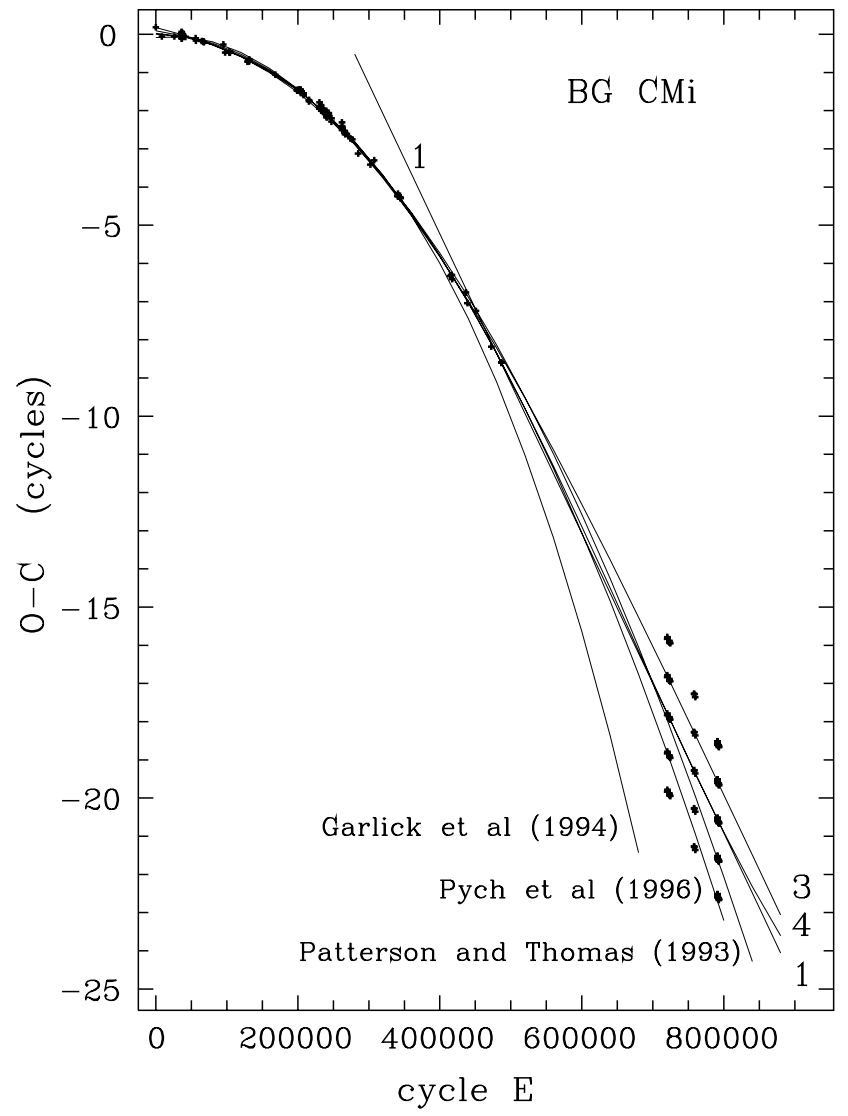

Fig. 9. O-C values for times of spin maxima calculated with the linear ephemeris (Pych et al. 1996) with a cycle numbering corresponding to the best 4th order polynomial fit. Fits are shown, which correspond to ephemerides published by Patterson \& Thomas (1993), Garlick et al. (1994) and Pych et al. (1996) for polynomials of order 2, 3 and 2, respectively. The order of our polynomial fits with optimal cycle counting are marked by numbers. Our parabolic fit is close to that of Pych et al. (1996) and is not shown separately. Because of possible cycle miscount after the gap, our data are shown with shifts of integer number of periods.

For reference, we have used again the linear part of the most recent ephemeris (3) published by Pych et al. (1996). The longterm variability of the period and thus the initial epoch is negligible for such a local fit, contrary to the complete interval.

In Fig. 9, different models of O-C variations are shown. We checked the ephemeris by Pych et al. (1996). Despite it satisfactory fits to the data obtained in 2002-03 and 2005, the spin maxima in 2003 occurred out of phase, so such a fit is not acceptable. The ephemeris by Patterson \& Thomas (1993) leads to a shift of the cycle numbering of all our data by unity, thus has the same problem with the year 2004. All our data may be well fitted by the ephemeris by Garlick et al. (1994) assuming a numbering shift of 7 cycles. However, it badly fits the data by Pych et al. (1996). Thus no fit from previously published ones describes the whole data set.

After such a long gap of 6 years, the main problem is the correct cycle numbering. For this purpose, we have determined the best values for separate seasons, using the maxima timings from Table 5, and the preliminary cycle numbering from the ephemeris by Pych et al. (1996). They are listed in Table 6. 
Table 5. Times of mean maxima from the multi-sinusoidal fit of individual runs of observations of BG CMi.

\begin{tabular}{ccccc}
\hline \hline$E$ & $t_{\max }-2400000$ & $\phi_{5}$ & $\phi_{\text {Pych }}$ & Rem \\
\hline 720253 & $52635.32067(47)$ & -0.05 & -17.83 & $V$ \\
720535 & $52638.30276(22)$ & 0.01 & -17.78 & $V$ \\
723524 & $52669.90440(37)$ & 0.03 & -17.88 & $W$ \\
723608 & $52670.79209(19)$ & -0.00 & -17.92 & $W$ \\
723898 & $52673.85825(15)$ & 0.01 & -17.92 & $W$ \\
724460 & $52679.80003(21)$ & 0.01 & -17.95 & $W$ \\
758066 & $53035.10202(20)$ & 0.02 & -19.27 & $V$ \\
758169 & $53036.19093(21)$ & 0.02 & -19.28 & $V$ \\
759761 & $53053.02230(18)$ & 0.00 & -19.36 & $V$ \\
790981 & $53383.09798(78)$ & -0.04 & -20.60 & $R$ \\
790981 & $53383.09882(777)$ & 0.04 & -20.52 & $V$ \\
791080 & $53384.14518(57)$ & 0.01 & -20.56 & $V$ \\
791080 & $53384.14560(67)$ & 0.05 & -20.52 & $R$ \\
791262 & $53386.06915(36)$ & -0.01 & -20.59 & $R$ \\
791262 & $53386.06917(37)$ & -0.01 & -20.58 & $V$ \\
792203 & $53396.01793(10)$ & -0.02 & -20.62 & $V$ \\
792203 & $53396.01806(10)$ & -0.01 & -20.61 & $R$ \\
793057 & $53405.04680(43)$ & -0.03 & -20.67 & $V$ \\
793057 & $53405.04708(44)$ & -0.00 & -20.64 & $R$ \\
\hline
\end{tabular}

Table 6. Seasonal ephemerids for spin maxima of BG CMi according to the linear fit $t_{E}=T_{0}+P \cdot\left(E-E_{0}\right)$.

\begin{tabular}{ccrc}
\hline \hline$T_{0}-2400000$ & $P$ & \multicolumn{1}{c}{$\phi_{5}$} & $E_{0}$ \\
\hline $52661.32970(11)$ & $0.010572652(66)$ & 0.001 & 722713 \\
$53041.43489(2)$ & $0.010572451(28)$ & 0.013 & 758665 \\
$53390.87981(9)$ & $0.010572466(116)$ & -0.003 & 791717 \\
$53105.31448(6)$ & $0.0105725772(20)$ & 0.020 & 764707 \\
\hline
\end{tabular}

The weighted mean value had allowed us to obtain a corrected difference of the cycle numbers between the initial epochs for the first (2002-03) and last (2005) seasons.

Assuming the new linear ephemeris for all our data (Table 6), one has to change the initial ephemeris by Pych et al. (1996). Assuming the quadratic model for O-C, from the values at the period $E=0$ and at $E=764707$ (our epoch for 2002-2004), we get $Q=-2.71(2) \times 10^{-13}$, significantly different from the value published by Pych et al. (1996). This shows that the "global" parabolic fit for all data is not satisfactory. Assuming an additional cubic term $Q_{3} E^{3}$ with fixed values of other 3 parameters, the value of $Q_{3}=+9.7 \times 10^{-20}$ had been estimated assuming that, for a negative $\dot{P}$, the second derivative $\ddot{P}$ is positive. This indicates a deceleration of the spin-up of the white dwarf. This result is based only on the analysis of period values at the beginning and end of known data, and is not dependent on possible minor cycle miscounts.

Our linear ephemeris fits well the last timings of Pych et al. (1996), so formally one may apply a composite "spline-type" model for the $\mathrm{O}-\mathrm{C}$ with parts of spin-up and constant periods. It resembles the "asymptotic parabola" model (Marsakova \& Andronov 1996) for extrema timings. Such abrupt period changes are often observed in classical eclipsing binary stars (e.g. Kreiner et al. 2001). Another approach is to apply highorder polynomials (e.g. Kalimeris et al. 1994).
For such polynomial models, we have chosen a free parameter which corresponds to the cycle number $E_{\mathrm{f}}$ of one of the minima from our sample. We have chosen the initial epoch for the last season 2005. The initial cycle number was chosen to correspond to the quadratic ephemeris by Pych et al. (1996), i.e. 791717 . Then it was modified within limits of \pm 10 cycles. For each trial value of $E_{\mathrm{f}}$ and of the degree of model, the following iterations were made. At first, this point replaced all our 19 timings, so giving high weight for this point. The computed best fit polynomial ephemeris has been used to determine the cycle numbers of all 19 timings. The numbering of the previous 90 points remained the same. Then a final polynomial fit was obtained. The test function was the rms deviation of the phases from the fit $\sigma_{\mathrm{O}-\mathrm{C}}$.

For the parabolic fit, the numbering remained the same, showing unique deep minimum at the $\sigma_{\mathrm{O}-\mathrm{C}}\left(E_{\mathrm{f}}\right)$ dependence at $E_{\mathrm{f}}=791717$. However, the maximal deviations exceded $0.4 P$, thus the fit is not acceptable. The cubic fit has an optimal solution for $E_{\mathrm{f}}=791714$, which corresponds to the ephemeris

$$
\begin{aligned}
\text { Max.HJD }= & 2445020.2791(3)+0.0105730179(37) E \\
& -5.28(13) \times 10^{-13} E^{2}+2.10(10) \times 10^{-13} E^{3} .
\end{aligned}
$$

However, the 4th order polynomial for $E_{\mathrm{f}}=791715$ gives much better fit to our data, with a scatter smaller by 1.5 times than that corresponding to the cubic fit. Thus it is the statistically optimal fit. The corresponding ephemeris is presented in the abstract. However, the "spline-type" and polynomial models may also not be ruled out.

For a final determination of the cycle numbering and choice of the appropriate model, one needs to analyse the spin maxima obtained between 1996 and 2002. However, the results of these observations are not published yet.

Future new observations will be crucial for the study of the rotational evolution of the white dwarf in this system, as the ephemerides obtained using different models will diverge in the next half-year or more.

\section{Conclusions}

- The ephemeris for the orbital minima has been corrected.

- The amplitude of spin variability has an abrupt maximum at the phase of the orbital dip after the mid-eclipse.

- The orbital light curves in $V$ and $R$ differ, with the color index having its miminimum (highest temperature) at the phase 0.42 and maximum at the phase 0.82 . The statistically optimal degree of the trigonometric polynomial is 2 , unlike the previously published value 6 .

- The spin variations in both colors have the same effective amplitudes and colors, contrary to the non-magnetic cataclysic variables with either superhumps or total eclipses and to the classical polars.

- The ephemeris for the spin maxima in 2002-2005 has been determined.

- The rate of the spin-up of the white dwarf has been significantly decreased as compared with previous years.

- The optimal mathematical model for the spin ephemeris corresponds to a fourth-order polynomial. However, 
because of the 6-year gap after the previously published observations, other cycle numbering over the gap may not be excluded. It may correspond either to the cubic ephemeris or to the "spline-like" ephemeris with the spin-up replaced by a constant period rotation.

- The obtained parameters may be used for comparison with theoretic models of accretion in magnetic cataclysmic variables. The further monitoring is needed for studies of rotational evolution of the white dwarf.

Acknowledgements. The authors are grateful to Jerrold L. Foote (CBA Utah) for sending us his unpublished data, This work was supported by the Korea Astronomy Observatory and Space Science Institute Research Fund 2003 and was partially supported by the Ministry of Education and Science of Ukraine.

\section{References}

Allen, C. W. 1973, Astrophysical Quantities (The Athlone Press)

Andronov, I. L. 1994, Odessa Astron. Publ., 7, 49 http://il-a.pochta.ru//oap7_049.ps.z

Andronov, I. L. 1997, A\&AS, 125, 207

Andronov, I. L. 2003, ASP Conf. Ser., 292, 391

Andronov I.L., Arai K., Chinarova L.L., et al., 1999, AJ., 117, 574

Andronov, I. L., \& Baklanov, A. V. 2004, Astron. School Rep., 5, 264

Andronov, I. L., Kim, Y. G., Shin, J.-H., \& Jeon, Y. B. 2005a, ASP Conf. Ser., 335, 355

Andronov, I. L., Ostrova, N. I., \& Burwitz, V. 2005b, ASP Conf. Ser., 335,229

Augusteijn, T., Schwarz, H. E., \& van Paradijs, J. 1991, A\&A, 247, 64

Chinarova, L. L. 1998, in Proc. 29th Conf. Variable Star Res., ed. J. Dusek, \& M. Zejda, Brno, Czech Republic, 38

Chochol, D., Andronov, I. L., Arkhipova, V. P., et al. 1999, CoSka, 29, 31

de Martino, D., Mouchet, M., Bonnet-Bidaud, J. M., et al. 1995, A\&A, 298,849

Foote, J. L. 2003, www . scopecraft . com/Text/

Observatory/CBA.htm
Garlick, M. A., Rosen, S. R., Mittaz, J. P. D., et al. 1994, MNRAS, 267,1095

Hellier, C. 1999, ApJ, 519, 324

Hellier, C. 2001, Cataclysmic Variable Stars. How and why they vary (Berlin: Springer)

Henden, A. A., \& Honeycutt, R. K. 1995, PASP, 107, 324

Johnson, H. L. 1955, Ann. d'Ap., 18, 292

Kalimeris, A., Rovithis-Livaniou, H., \& Rovithis, P. 1994, A\&A, 282, 775

Kim, Y. G., Andronov, I. L., \& Jeon, Y. B. 2004, J. Astron. Space Sci., 21(3), 191, http://ksss . or . kr/dtp/J200409/kimyonggi .ps

Kim, Y., \& Beuermann, K. 1995, A\&A, 298, 165

Kim, Y., \& Beuermann, K. 1996, A\&A, 307, 824

Kreiner, J. M., Kim, C.-H., \& Nha, I.-S. 2001, An Atlas of O-C Diagrams of Eclipsing Binary Stars, Cracow, Poland

Massey, P., \& Davis, L. E. 1992, A User's Guide to Stellar CCD Photometry with IRAF

Marsakova, V. I., \& Andronov, I. L. 1996, Odessa Astron. Publ., 9, 127, http://oap09. pochta.ru

McHardy, I. M., Pye, J. P., Fairall, A. P., et al. 1984, MNRAS, 210, 663

Norton, A. J., McHardy, I. M., Lehto, H. J., \& Watson, M. G. 1992, MNRAS, 258, 697

Ostrova, N. I., Shugarov, S. Yu., \& Andronov, I. L. 2005, Ap\&SS, 296, 473

Patterson, J. 1994, PASP, 106, 209

Patterson, J., \& Thomas, G. 1993, PASP, 105, 59

Penning, W. R., Schmidt, G. D., \& Liebert, J. 1986, ApJ, 301, 881

Pych, W., Semeniuk, I., Olech, A., \& Ruszkowski, M. 1996, AcA, 46, 279

Singh, J., Agrawal, P. C., Apparao, K. M. V., Vivekananda Rao, P., \& Sarma, M. B. K. 1991, ApJ, 380, 208

Shakhovskoj, N. M., \& Kolesnikov, S. V. 1997, IAUC, 6760

Szkody, P., \& Brownlee, D. E. 1977, ApJ, 212, L113

Tremko, J., Andronov, I. L., Chinarova, L. L., et al. 1996, A\&A, 312, 121

Walker, M. F. 1954, PASP, 66, 71

Warner, B. 1986, MNRAS, 219, 347

Warner, B. 1995, Cataclysmic Variable Stars (Cambridge Univ. Press) Wynn, G. A., \& King, A. R. 1992, MNRAS, 255, 83 